\title{
Programming human pluripotent stem cells into white and brown adipocytes
}

\section{Citation}

Ahfeldt, Tim, Robert T. Schinzel, Youn-Kyoung Lee, David Hendrickson, Adam Kaplan, David H. Lum, Raymond Camahort, et al. 2012. "Programming Human Pluripotent Stem Cells into White and Brown Adipocytes." Nature Cell Biology 14 (2) (January 15): 209-219. doi:10.1038/ncb2411.

\section{Published Version}

doi:10.1038/ncb2411

\section{Permanent link}

http://nrs.harvard.edu/urn-3:HUL.InstRepos:14068403

\section{Terms of Use}

This article was downloaded from Harvard University's DASH repository, and is made available under the terms and conditions applicable to Other Posted Material, as set forth at http:// nrs.harvard.edu/urn-3:HUL.InstRepos:dash.current.terms-of-use\#LAA

\section{Share Your Story}

The Harvard community has made this article openly available.

Please share how this access benefits you. Submit a story.

\section{Accessibility}




\title{
Programming human pluripotent stem cells into white and brown adipocytes
}

\author{
Tim Ahfeldt ${ }^{1,2,3,14}$, Robert T. Schinzel ${ }^{1,2,3,4,14}$, Youn-Kyoung Lee ${ }^{1,2,3,14}$, David \\ Hendrickson $^{1,5}$, Adam Kaplan ${ }^{1,2,3}$, David H. Lum ${ }^{6,7}$, Raymond Camahort ${ }^{1,2,3}$, Fang Xia ${ }^{1,2,3}$, \\ Jennifer Shay ${ }^{1,2,3}$, Eugene P. Rhee ${ }^{3,5}$, Clary B. Clish ${ }^{5}$, Rahul C. Deo ${ }^{8,9,10}$, Tony Shen ${ }^{1,2}$, \\ Frank H. Lau ${ }^{2,3}$, Alicia Cowley ${ }^{1,2}$, Greg Mowrer ${ }^{2,3}$, Heba Al-Siddiqi ${ }^{1,2,3,11}$, Matthias \\ Nahrendorf ${ }^{12}$, Kiran Musunuru ${ }^{1,5,13}$, Robert E. Gerszten ${ }^{3,5}$, John L. Rinn ${ }^{1,5}$, and Chad A. \\ Cowan ${ }^{1,2,3,5,15}$ \\ ${ }^{1}$ Department of Stem Cell and Regenerative Biology, Harvard University, Cambridge, \\ Massachusetts 02138, USA \\ ${ }^{2}$ Center for Regenerative Medicine, Massachusetts General Hospital, Boston, Massachusetts \\ 02114, USA \\ ${ }^{3}$ Cardiovascular Research Center, Massachusetts General Hospital, Boston, Massachusetts \\ 02114, USA \\ ${ }^{4}$ Institut für Biologie-Mikrobiologie, Fachbereich Biologie, Chemie, Pharmazie, Freie Universität \\ Berlin, Königin-Luise-Strasse 12-16, 14195 Berlin, Germany \\ ${ }^{5}$ Broad Institute, 7 Cambridge Center, Cambridge, Massachusetts 02142, USA \\ ${ }^{6}$ Huntsman Cancer Institute, University of Utah, Salt Lake City, Utah 84112, USA \\ ${ }^{7}$ Department of Oncological Sciences, University of Utah, Salt Lake City, Utah 84112, USA \\ ${ }^{8}$ Cardiovascular Research Institute, University of California San Francisco, San Francisco, \\ California 94143, USA \\ ${ }^{9}$ Department of Medicine, University of California San Francisco, San Francisco, California \\ 94143, USA \\ ${ }^{10}$ Institute for Human Genetics, University of California San Francisco, San Francisco, California \\ 94143, USA \\ ${ }^{11}$ Research Division, Qatar Foundation, Doha, Qatar \\ ${ }^{12}$ Center for Systems Biology, Massachusetts General Hospital and Harvard Medical School, \\ Simches Research Building, 185 Cambridge St., Boston, Massachusetts 02114, USA
}

${ }^{13}$ Department of Medicine, Brigham and Women's Hospital, Boston, Massachusetts 02115, USA

\author{
Abstract \\ (C) 2012 Macmillan Publishers Limited. All rights reserved. \\ ${ }^{15}$ Correspondence should be addressed to: C.A.C. (ccowan@fas.harvard.edu). \\ ${ }^{14}$ These authors contributed equally to this work. \\ Note: Supplementary Information is available on the Nature Cell Biology website \\ COMPETING FINANCIAL INTERESTS \\ The authors declare no competing financial interests. \\ Reprints and permissions information is available online at http://www.nature.com/reprints
}


The utility of human pluripotent stem cells is dependent on efficient differentiation protocols that convert these cells into relevant adult cell types. Here we report the robust and efficient differentiation of human pluripotent stem cells into white or brown adipocytes. We found that inducible expression of PPARG2 alone or combined with $C E B P B$ and/or PRDM16 in mesenchymal progenitor cells derived from pluripotent stem cells programmed their development towards a white or brown adipocyte cell fate with efficiencies of $85 \%-90 \%$. These adipocytes retained their identity independent of transgene expression, could be maintained in culture for several weeks, expressed mature markers and had mature functional properties such as lipid catabolism and insulin-responsiveness. When transplanted into mice, the programmed cells gave rise to ectopic fat pads with the morphological and functional characteristics of white or brown adipose tissue. These results indicate that the cells could be used to faithfully model human disease.

The derivation of human embryonic stem cells ${ }^{1}$ (hESCs) and generation of human induced pluripotent stem cells ${ }^{2,3}$ (hiPSCs) have made possible the creation of patient-specific in vitro cell models. As human pluripotent stem cells (hPSCs) self-renew and have the potential to differentiate into any adult cell type, they represent an inexhaustible supply of cells for studying normal cell function and disease pathogenesis. Although the number of patientderived hiPSC lines is rapidly increasing ${ }^{4,5}$, the principal obstacle for understanding disease remains the difficulty of differentiating hPSCs into adult cell types.

White adipose tissue, which is specialized for energy storage, is readily obtained from patients but is difficult to maintain and cannot expand in culture. Much of our insight into the differentiation and transcriptional regulation of adipocytes has come from the mouse cell line 3T3-L1, which can be differentiated into white adipocytes by exposure to a combination of factors ${ }^{6,7}$ and was used to identify the transcription factor peroxisome proliferatoractivated receptor $\gamma 2$ (PPARG2; ref. 8), widely regarded as a master regulator of adipogenesis 9 .

Brown adipose tissue uses the chemical energy in lipids and glucose to produce heat through non-shivering thermogenesis ${ }^{10}$. The recent discovery that adult humans have brown adipose depots of a size that is inversely correlated with body mass index ${ }^{11-13}$ has fuelled considerable interest in the therapeutic potential of brown adipocytes. The transcription factors encoded by CCAAT/enhancer-binding protein beta $(C E B P B)$ and PR domain containing 16 (PRDM16) are thought to function as key regulators of brown fat development and function ${ }^{14,15}$.

A number of groups have developed human-cell-based models for the study of adipogenesis using either mesenchymal stem cells (MSCs) from bone marrow or other tissues ${ }^{16,17}$ or adipose-derived stromal vascular cells ${ }^{18}$ (ADSVCs). Although these cellular systems have proved useful, they have limitations including limited proliferative potential, decreased differentiation with continued passaging ${ }^{19}$ and variable differentiation potential. To overcome these obstacles, several groups have sought to use hPSCs to generate human adipocytes, but reports so far have been limited to white adipocytes ${ }^{20-23}$. Moreover, the efficient generation of large numbers of hPSC-derived adipocytes, with detailed phenotypic characterization that documents fidelity to primary cells, has remained elusive. For hPSCs to be useful cellular models of adipose-related disease, it is necessary to develop reliable and scalable protocols for their differentiation into adipocytes. Here we report simple, consistent and highly efficient protocols to generate mature functional adipocytes-either white or brown-from hPSCs. 


\section{RESULTS}

\section{Differentiation of hPSCs to MPCs}

Several protocols for generating MSCs or mesenchymal progenitor cells (MPCs) from hPSCs have previously been described ${ }^{24,25}$. Notably, these cells had the potential to form adipocytes. We sought to simplify the derivation of MPCs from hPSCs (Fig. 1a and Supplementary Fig. S1A). Three hESC lines and two hiPSC lines ${ }^{26}$ were differentiated into embryoid bodies that after two days in suspension culture had a characteristic rounded shape with defined and smooth borders. After ten days, these embryoid bodies were plated to adherent cell culture dishes, and fibroblast-like cells were observed growing from the embryoid bodies (Supplementary Fig. S1B). We analysed the expression levels of pluripotency genes and mesoderm development genes at different stages during the differentiation protocol (Supplementary Fig. S1C). We observed a transient increase in expression of the mesendoderm marker goosecoid (GSC) during differentiation and the level of expression declined after prolonged culture of the fibroblast-like cells. The mesodermal marker T-box transcription factor 3 (TBX3) was absent in the pluripotent stage but was expressed during differentiation, and expression was maintained in cultured fibroblast-like cells. NANOG, a marker of pluripotency, was observed at very high levels in pluripotent cells but rapidly diminished during differentiation.

The derived fibroblast-like cells were replicative and were capable of expansion for 10-12 passages. We analysed these cells with flow cytometry for markers characteristic of an MSC fate-Stro1, CD29, CD105, CD73 and CD44-and negative control markers of the blood lineage-CD19 and CD4 (Fig. 1b and Supplementary Fig. S2A-D). We found that more than 96\% of the fibroblast-like cells expressed CD73, CD29, CD44 and CD105; ADSVCs similarly expressed CD73, whereas hPSCs lacked the CD73 surface antigen entirely. Global transcriptional analysis of the derived cells showed high similarity to a variety of MSC lines (Supplementary Fig. S3). We were able to differentiate the fibroblast-like cells into osteoblasts and chondrocytes (Supplementary Fig. S1D,E) and adipocytes (see below). In light of the characteristic markers and the trilineage differentiation results, we hereafter term the fibroblast-like cells MPCs.

\section{Differentiation of MPCs into white adipocytes}

To determine whether our hPSC-derived MPCs could generate white adipocytes as previously reported ${ }^{24,27}$, we examined their response to known inducers of adipogenesisinsulin, rosiglitazone and dexamethasone; for comparison we used human ADSVCs. After 21 days of exposure to a combination of these factors, a small percentage of MPCs and ADSVCs contained multilocular lipid droplets resembling those often found in immature white adipocytes (Fig. 2b, top panels and Supplementary Fig. S4A-D). ADSVC lines from distinct donors showed considerable variation in their ability to differentiate into white adipocytes, $10 \%-70 \%$, as has previously been reported ${ }^{28}$. The white adipocyte differentiation potential of MPC lines was also variable and was less than that of ADSVCs; only 3\%-10\% of MPCs differentiated into white adipocytes (Fig. 2a).

\section{Programming MPCs into white adipocytes}

We attempted to improve the differentiation of MPCs to white adipocytes with the temporally controlled expression of a programming factor, namely PPARG2, a key regulator of adipogenesis ${ }^{9,29-31}$. MPCs were transduced with a lentiviral construct with a doxycycline-inducible promoter driving PPARG2 complementary DNA expression (lentitet-PPARG2) and a construct constitutively expressing the reverse tetracycline transactivator $^{32}$ (lenti-rtTA) allowing controlled expression of PPARG2 (Fig. 1c). We determined that the transduction efficiency of MPCs was approximately $98 \%$ (s.d. $\pm 1 \%$ ) as 
measured by the proportion of GFP-positive cells following infection with lentiviral concentrations of greater than 375 copies per cell (Supplementary Fig. S5A,B). MPCs or ADSVCs were cultured in adipogenic media (differentiated) and either transduced (programmed) or not (unprogrammed) with lenti-tet-PPARG2 and lenti-rtTA (ref. 32) in the presence of doxycycline for 16 days, after which the doxycycline was removed from the media and the cells were cultured for an additional five days (Fig. 1a) to generate transgeneindependent adipocytes. After 21 days, we consistently observed that most PPARG2programmed, differentiated MPCs had the typical morphology of mature white adipocytes, with a single large, well-defined lipid droplet, in contrast to unprogrammed, differentiated MPCs (Fig. 2a-c).

To determine the efficiency of white adipocyte differentiation, hPSC-derived adipocytes were immunostained with an antibody against CCAAT/enhancer-binding protein alpha (CEBPA; Fig. 2a). By counting the number of CEBPA-positive nuclei, we determined that on average $88 \%$ of PPARG2-programmed, differentiated MPCs were white adipocytes, compared with $9 \%$ of unprogrammed, differentiated MPCs $(n=3)$. We immunostained programmed and unprogrammed MPCs at day 21 of differentiation with antibodies against fatty-acid-binding protein 4 (FABP4), PPARG2 and CEBPA (Fig. 2b,c). We observed strong staining of the cytoplasm with antibodies against FABP4 and nuclear staining with antibodies against PPARG2 and CEBPA. The cells were also stained with BODIPY and had a morphology characteristic of mature white adipocytes, with most positively stained cells containing one large, predominant lipid droplet and a few surrounding small droplets. To confirm that the hPSC-derived white adipocytes were not dependent on transgene expression, we cultured the cells for up to four additional weeks in the absence of doxycycline. Twenty days after doxycycline withdrawal, these cells maintained the morphologic appearance of mature adipocytes, incorporated BODIPY, stained positive for CEBPA and exhibited minimal expression of lentivirus-specific PPARG2 (Supplementary Fig. S4E). Furthermore, we investigated the kinetics of PPARG2-programmed differentiation into adipocytes. To that end we differentiated the cells for 21 days and withdrew doxycycline at different time points and examined the gene expression of adipogenic markers by quantitative polymerase chain reaction with reverse transcription (RT-qPCR; Supplementary Fig. S8B). For the markers encoded by hormone-sensitive lipase $(H S L)$ and adiponectin $(A D I P O Q)$, a plateau was reached after 14 days of doxycycline administration. FABP4 showed the highest level of expression after 16 days of doxycycline administration. Thus, our data indicate that a 16-day pulse of PPARG2 expression is sufficient to permanently switch the fate of MPCs to the white adipocyte lineage.

\section{Programming MPCs into brown adipocytes}

We extended our programming approach to achieve differentiation of MPCs into brown adipocytes. PRDM16 has previously been shown to convert murine myoblasts into brown adipocytes $^{33}$, and a combination of CEBPB and PRDM16 to convert mouse cells and human fibroblasts into adipocytes with brown adipocyte characteristics ${ }^{15}$. Accordingly, to generate brown adipocytes, we transduced MPCs with combinations of doxycycline-inducible lentiviral constructs (Supplementary Fig. S5D) encoding the transcription factors PPARG2, CEBPB and PRDM16 and differentiated the cells in doxycyline-containing adipogenic medium. Generally, doxycycline was removed from the media after 14 days in culture and the cells were cultured for an additional 7 days (Fig. 1a). Three transcription factor combinations showed a statistically significant increase in the level of expression of uncoupling protein 1 ( $U C P 1 ; n=3$; Supplementary Fig. S5E). This included the previously reported transcription factor combination CEBPB-PRDM16 as well as PPARG2-CEBPB and PPARG2-CEBPB-PRDM16. We noted that combinations that included PPARG2 showed a much greater induction of $U C P 1$. We also found that the expression of $A D I P O Q$ 
and cell-death-inducing DFFA-like effector c (CIDEC) was similar among the three transcription factor combinations and, notably, lower than that seen in white adipocytes programmed with PPARG2 alone (Supplementary Fig. S5E). On the basis of these observations, we chose to focus further work on two of the transcription factor combinations, PPARG2-CEBPB and PPARG2-CEBPB-PRDM16.

After 21 days, we observed striking morphological differences between PPARG2programmed (white) adipocytes and PPARG2-CEBPB-programmed or PPARG2-CEBPBPRDM16-programmed (brown) adipocytes; programmed brown adipocytes generally had a multilocular lipid droplet morphology, and very few cells had the monolocular appearance noted in programmed white adipocytes (Fig. $2 \mathrm{~b}-\mathrm{d}$ ). We stained programmed brown adipocytes for UCP1 and noted strong staining of the cytoplasm, with almost no staining in unprogrammed cells. We further stained programmed brown adipocytes with the mitochondrial marker MitoTracker; we observed strong staining in the cytoplasm, with a more diffuse morphology and much less staining in unprogrammed cells (Fig. 2d). These findings were consistent with a brown adipocyte cell fate and distinguished the programmed brown adipocytes from white adipocytes.

\section{hPSC-derived adipocytes express mature markers}

We carried out RT-qPCR for a panel of adipocyte marker genes, including lipoprotein lipase (LPL), HSL, ADIPOQ, FABP4, CEBPA and PPARG2 (Supplementary Table S1), in undifferentiated MPCs and ADSVCs and in unprogrammed or PPARG2-programmed, differentiated cells ( $n=3$; Fig. 3a and Supplementary Fig. S8A). Adipocyte marker gene expression was absent in the undifferentiated cell lines with the exception of PPARG2, which was detected in ADSVCs as previously reported ${ }^{34,35}$. We found low levels of expression of these genes in unprogrammed, differentiated MPCs and ADSVCs. In contrast, PPARG2-programmed, differentiated MPCs and ADSVCs at day 21 exhibited high levels of expression of all of the adipocyte marker genes, consistent with a mature white adipocyte fate.

We next compared the expression signature of PPARG2-programmed white adipocytes with PPARG2-CEBPB-programmed and PPARG2-CEBPB-PRDM16-programmed brown adipocytes ( $n=3$; Fig. $3 \mathrm{~b}$ ). The level of gene expression was similar for the endogenous $P P A R G 2$ gene and $L P L$. As expected, programmed white adipocytes showed a higher level of expression of the white adipocyte markers $A D I P O Q, H S L$ and FABP4 when compared with programmed brown adipocytes; programmed brown adipocytes showed a higher level of expression of the brown adipocyte markers $U C P 1$ and elongation of very long chain fatty acids like-3 (ELOVL3), and peroxisome proliferator-activated receptor $\gamma 1-\mathrm{a}(P G C 1 A)$, a major regulator of mitochondrial biogenesis, as well as the mitochondrial marker cytochrome $c_{1}$ (CYC1; ref. 36). Notably, these results were independent of transgene expression (Supplementary Fig. S5C), confirming that the adipocyte markers observed were the result of an endogenous cellular program.

\section{Programmed white adipocytes have a transcriptional signature similar to primary adipocytes}

To evaluate how closely our hPSC-derived white adipocytes resembled primary white adipocytes on a genome-wide scale, we generated and analysed transcript profiles from undifferentiated and PPARG2-programmed, differentiated ADSVCs and MPCs in addition to primary fat on two independent array platforms (Fig. 4a,b and Supplementary Fig. S6AD). Both platforms yielded highly concordant results using a significance analysis of microarrays (SAM) algorithm to rank genes present on both platforms (Spearman rank correlation $=0.84)$. Furthermore, overlap of differentially expressed genes at a $5 \%$ false 
discovery rate (FDR) between both platforms was extremely high $(P<10-300$, hypergeometric test). For the following analyses and figures, we will focus on the Affymetrix data. Unsupervised clustering by the Pearson correlation coefficient for all of the expressed genes $(\sim 20,000)$ resulted in two primary clusters: PPARG2-programmed white adipocytes, derived from both MPCs and ADSVCs, and primary white fat $(r=0.95)$; and undifferentiated ADSVCs and MPCs $(r=0.88)$. To determine the most significant changes in gene expression between the programmed/primary adipocytes and undifferentiated populations (ADSVCs and MPCs) we used the SAM algorithm to call 2,136 differentially expressed genes using a 5\% local FDR (Fig. 4a; ref. 37). We carried out Gene Ontology (GO) term analysis using the DAVID Bioinformatics Resource 38,39 and found that the most prominently enriched gene sets in the 2,136 differentially expressed genes included annotations for PPARG2 signalling pathways as well as metabolism and genesis of fatty acids, and lipids ( $P<10^{-6}, 10^{-9}$ and $10^{-10}$, respectively; Supplementary Table S2). We further analysed the genes that showed the greatest differences between PPARG2programmed adipocytes and primary fat using the SAM algorithm and GO terms and observed that many of these genes could be attributed to the various non-adipocyte cell types present in adipose tissue, including red blood cells, leukocytes, vascular endothelium and mesenchymal cells (Supplementary Table S3). Last, we examined a panel of adipocytespecific genes and found that the level of expression was higher in the programmed/primary white adipocytes in comparison with the undifferentiated cells (Fig. 4b). Overall, the striking similarity of the primary white fat samples with the programmed white adipocytes $(r=0.95)$ indicates that PPARG2 induction is a reliable and reproducible methodology for the derivation of homogeneous adipocytes from different hPSC types.

\section{Programmed brown adipocytes have a distinct signature from white adipocytes}

To assess our programmed brown adipocytes at the transcrip-tome level, we generated and analysed transcript profiles from PPARG2-CEBPB-programmed and PPARG2-CEBPBPRDM16-programmed adipocytes and compared these with transcript profiles from primary white adipose tissue in addition to undifferentiated and PPARG2-programmed, differentiated ADSVCs and MPCs (Supplementary Fig. S6E). Hierarchical clustering with Pearson's correlation of 2,869 differentially expressed genes revealed that all of the adipose samples (programmed brown, programmed white and primary white) co-segregate separately from the undifferentiated samples (ADSVCs and MPCs; $r=0.86$ ). Importantly, within the adipose cluster, the programmed brown adipocytes $(r=0.95)$ cluster distinctly from the programmed and primary white adipose $(r=0.90)$.

We then focused on a panel of genes that are associated with brown fat or white fat identity ${ }^{36,40,41}$ (Fig. 4c). All of the genes that have been described as brown adipose tissue markers (UCP1, CYC1, NDUFA11, NDUFA13, CMT1A, ELOVL3, DIO2, LHX8, COX8A and $C Y F I P 2$ ) show the highest level of expression in programmed brown adipocytes, with the only outlier being endogenous PRDM16, which showed the highest level of expression in primary and programmed white adipocytes. All differentiated cells expressed general adipocyte markers (CIDEC, PLIN1 and FABP4) at much higher levels than undifferentiated cells. Genes associated with white fat identity ( $D P T$ and $I N H B B$ ) were most highly expressed in programmed and primary white adipocytes.

To confirm the low level of expression of PRDM16 in programmed brown adipocytes, we carried out RT-qPCR using a primer that detects only endogenous PRDM16 as well as a primer that detects viral and endogenous PRDM16. We found no obvious differences in endogenous PRDM16 expression between differentiated untransduced MPCs and PPARG2CEBPB- or PPARG2-CEBPB-PRDM16-programmed adipocytes (Supplementary Fig. S8F). Endogenous PRDM16 was almost undetectable in pluripotent control cells. Untransduced MPCs, PPARG2-CEBPB- or PPARG2-CEBPB-PRDM16-programmed 
adipocytes were analysed at day 5 and day 25 of differentiation. In all conditions, endogenous PRDM16 was expressed at day 5 of differentiation and showed an increase in the level of expression at day 25. Endogenous PRDM16 expression was comparable between untransduced and programmed cells. Next we used a primer that detects viral and endogenous PRDM16 expression. At day 5 of differentiation in medium containing doxycyline, we noted a 40-fold increase in the level of PRDM16 expression in the PPARG2-CEBPB-PRDM16-programmed adipocytes. Doxycycline was removed from the medium and the PRDM16 expression dropped to comparable low levels in all conditions. Thus, endogenous PRDM16 expression is not necessary for the differentiation of hPSCderived MPCs into brown adipocytes. It is possible that the PRDM16 expression that we noted in untransduced differentiated MPCs is sufficient to govern brown adipocyte differentiation or that the differentiation does not rely on PRDM16 in our human in vitro system.

\section{Programmed white adipocytes exhibit mature functional properties}

To investigate the functional capabilities of hPSC-derived white adipocytes, we measured a key property of mature white adipocytes, the ability to carry out lipolysis, through the release of glycerol after exposure to the $\beta$-adrenergic agonist isoproterenol in undifferentiated ADSVCs and programmed or unprogrammed, differentiated ADSVCs and MPCs ( $n=3$; Fig. 5a). We found that only the programmed, differentiated cells responded to isoproterenol treatment with the breakdown of triglycerides and release of glycerol (ADSVCs, $P=0.01$; MPCs, $P=0.002$ ). We also assessed the secretion of the hormone adiponectin, which regulates glucose and fatty acid catabolism and is produced by adipocytes ${ }^{42,43}$, using an enzyme-linked immunosorbent assay (ELISA). High levels of secreted adiponectin were observed from all PPARG2-programmed cell lines, including several differentiated hPSC-derived MPC lines, with low levels from control cell lines $(n=$ 3; Fig. 5c). Using a similar ELISA assay, we observed low levels of leptin secretion (25 pg $\mathrm{ml}^{-1}$ ) into the medium from PPARG2-programmed cells (Supplementary Fig. S8C).

We characterized de novo fatty acid synthesis and storage in hPSC-derived adipocytes using a tandem mass spectroscopy lipidomics platform ${ }^{44}$. We profiled the cellular lipid content of ADSVC- and hPSC-derived white adipocytes programmed with PPARG2 expression and compared the findings with lipid profiles obtained from primary human white fat (Fig. 5c). Spearman correlation coefficients (SCC) were analysed across all triglyceride species, and PPARG2-programmed, differentiated cells were much more similar to primary white adipocytes (SCC 0.75-0.87) than either unprogrammed, differentiated cells (SCC 0.410.62) or undifferentiated cells (SCC 0.21-0.32; Supplementary Fig. S7). Consistent with increased expression levels of genes involved in de novo fatty acid synthesis, we observed higher levels of some short-chain fatty acids in programmed white adipocytes than in primary white adipose (Supplementary Fig. S7A-C). The diacylglycerol content of programmed white adipocytes was also slightly altered as compared with primary white adipose tissue (Supplementary Fig. S7D), which could be the result of either de novo synthesis or enhanced lipolysis. Membrane lipids, such as phosphatidylcholines, were similar in all cell types analysed (Supplementary Fig. S7E). Overall, we observed remarkable congruence among the lipid profiles of programmed white adipocytes and primary white adipose tissue, especially with respect to triglycerides, which represent the most abundant lipids in mature white adipocytes.

Finally, we assessed whether the programmed white adipocytes respond to insulin in a manner similar to mature primary white adipocytes. We analysed insulin signalling of programmed white adipocytes in the absence and presence of free fatty acids (FFA), an established contributor to induce insulin resistance in white adipocytes ${ }^{45}$. We examined insulin response by assessing protein expression levels of AKT and phosphorylated AKT 
(pAKT(Ser 473); Fig. 5d). In the basal starved state, no pAKT(Ser 473) product was detected; administration of insulin robustly upregulated pAKT(Ser 473). Co-administration of FFA decreased the level of insulin-induced phosphorylation of AKT, demonstrating that the hPSC-derived adipocytes appropriately model FFA-induced insulin resistance. We also analysed the uptake of $\left[{ }^{3} \mathrm{H}\right] 2$-deoxy-d-glucose (Fig. 5e) and found that the basal level of glucose uptake was similar between PPARG2-programmed and unprogrammed cells. After exposure to insulin, a significant increase in the level of glucose uptake was noted in both cell types ( $P=0.05$ for unprogrammed cells, $P=0.0007$ for programmed cells) but was substantially higher in the PPARG2-programmed cells $(P=0.009)$. Taken together, these results validate programmed white adipocytes as a model of insulin response and insulin resistance.

\section{Programmed brown adipocytes exhibit mature functional properties}

The characteristic function of brown adipocytes is thermogenesis, driven by the catabolic breakdown of lipids. We therefore measured the release of glycerol from our various cells to determine the extent of lipolysis on exposure to forskolin. As expected, both white and brown hPSC-derived adipocytes exhibited a significantly higher level of glycerol release in response to forskolin treatment (Fig. 6a) when compared with unprogrammed cells. For the programmed brown adipocytes, we further examined the response to the $\beta$-adrenergic agonist isoproterenol and observed a significant increase in the level of glycerol release when compared with unprogrammed cells (Supplementary Fig. S8D).

To further distinguish between white and brown adipocytes at a functional level, we extended our characterization to the mitochondrial function of our cells. We analysed the oxygen consumption rate (OCR) and extracellular acidification rate (ECAR) using an extracellular flux analyser (Fig. 6b,c); the basal OCR and ECAR rates were highest in the programmed brown adipocytes. We then injected compounds that modulate mitochondrial function sequentially and measured the effect on OCR and ECAR after the addition of each compound. We first administered oligomycin to determine ATP turnover and the degree of proton leak. At the baseline, the programmed brown adipocytes showed slightly elevated levels of proton leak when compared with unprogrammed cells. After the addition of the electron transport chain decoupler carbonyl cyanide-m-chlorophenylhydrazone (CCCP), we measured the maximal respiratory capacity. Programmed brown adipocytes showed significantly higher levels of OCR and ECAR when compared with the unprogrammed cells, whereas programmed white adipocytes did not. Finally, we administered antimycin to inhibit the flux of electrons through complex III and prevent oxygen consumption by the cytochrome $c$ oxidase in the mitochondria. The OCR for all cell types dropped to postoligomycin levels. Taken together, these results confirm that programmed brown adipocytes have a significantly increased level of mitochondrial activity, an important functional characteristic of brown adipose tissue.

\section{In vivo transplantation of hPSC-derived adipocytes}

We subcutaneously administered hPSC-derived brown and white adipocytes, as well as control 3T3-F442A cells, to immunocompromised $\mathrm{Rag}^{-/-} \mathrm{II} 2 \mathrm{rg}^{-/-}$mice to study the function of the cells in vivo. The transplants were collected and histologically examined after 4-6 weeks; importantly, no teratomas were observed $(n=20)$. We confirmed the extracorporeal origin of the collected cells by immunohistochemical staining with MAB1281, a human-specific nuclear antibody that does not stain nuclei in mouse cells (Fig. 7a,b and Supplementary Fig. S8E). Programmed white adipocytes stained positively for the adipocyte marker CEBPA, whereas programmed brown adipocytes were positive for UCP1 (Fig. 7a,b) in adjacent slides. Transplanted programmed white and brown adipocytes had a morphology characteristic of primary adipocytes. Finally, we assessed the white and brown 
adipocyte transplants by fluordeoxyglucose $\left({ }^{18} \mathrm{FDG}\right)$ uptake followed by positron-emission tomography-computed tomography (PET-CT), a technique that has been used to detect brown adipose tissue in adult humans ${ }^{11,13,46,47}$. Consistent with brown adipocytes acting as a 'glucose sink', the transplanted hPSC-derived brown adipocytes were highly FDG-avid and exhibited a clear PET signal when compared with hPSC-derived white adipocytes (Fig. $7 c)$.

\section{DISCUSSION}

We have established efficient protocols to generate white and brown adipocytes from hPSCs. Brown adipocytes show distinct morphology and gene expression profiles from white adipocytes, and each type of cell demonstrated functional properties that are characteristic of the corresponding tissue types in vivo. Transplantation of the cells into mice yielded tissues with functional and morphological similarities to primary white and brown adipose tissues. Taken together, these experiments confirm the identity and maturity of the hPSC-derived white and brown adipocytes and indicate that the cells could be used to faithfully model human disease.

\section{METHODS}

Methods and any associated references are available in the online version of the paper at http://www.nature.com/naturecellbiology

\section{METHODS}

\section{Maintenance of pluripotent cells, generation of MPCs and adipocyte differentiation}

hESCs and hiPSCs were cultured feeder free on Geltrex (Invit rogen) in the chemically defined medium mTESR1 (Stem Cell Technologies). To induce differentiation of hESCs and hiPSCs into embryoid bodies, hPSCs were disaggregated with dispase into small clumps containing 5-10 cells and transferred to low-adhesion plastic 6-well dishes (Costar Ultra Low Attachment; Corning Life Sciences) in growth medium containing DMEM, 15\% FBS and $1 \%$ GlutaMAX After 7 days in suspension culture, embryoid bodies were collected and replated on gelatin-coated 6-well dishes in medium containing DMEM, 10\% FBS and 1\% GlutaMAX. After cells reached confluency (in approximately 5 days), they were trypsinized $(0.25 \%$ trypsin) and replated on cell culture dishes containing MPC growth medium containing DMEM, 15\% FBS, $1 \%$ GlutaMAX and $2.5 \mathrm{ng} \mathrm{ml}^{-1}$ bFGF (Aldevron). Cells were passaged with a 1:3 split ratio and used for differentiation experiments before passage 8. Adipogenic differentiation was carried out for 21 days using adipogenic differentiation medium containing DMEM, 7.5\% knockout serum replacement (KOSR; Invitrogen), 7.5\% human plasmanate, $0.5 \%$ non-essential amino acids, $1 \%$ penicillin and streptomycin, $0.1 \mu \mathrm{M}$ dexamethasone, $10 \mu \mathrm{g} \mathrm{ml}^{-1}$ insulin (Sigma) and $0.5 \mu \mathrm{M}$ rosiglitazone. Adipogenic differentiation medium was supplemented for 16 days with doxycyline $\left(700 \mathrm{ng} \mathrm{ml}^{-1}\right)$, and afterwards cells were maintained in culture in the absence of doxycycline until day 21 or longer as experiments required.

\section{Derivation and maintenance of ADSVCs}

Primary human adipose tissue was obtained from surgical waste of patients who had undergone elective surgery Adipose was digested with Liberase (Roche) for $1 \mathrm{~h}$ with gentle shaking at $37^{\circ} \mathrm{C}$ Digested tissue was forced through a $250 \mu \mathrm{m}$ filter, and the filtrate was collected and centrifuged. The resulting stromal vascular cell pellet was washed twice with PBS and plated onto gelatin-coated plates $(0.1 \%)$ in ADSVC growth media (DMEM, 10\% 
FBS, $1 \%$ penicillin and streptomycin and $2.5 \mathrm{ng} \mathrm{ml}^{-1} \mathrm{bFGF}$ (Aldevron)). ADSVCs were passaged using trypsin on reaching confluency.

\section{Inducible transgenes}

To generate pLV-tetO-attR1/R2, pLV-tetO (ref. 48) was linearized with EcoRI followed by treatment with DNA polymerase I, large (Klenow) fragment to fill in and blunt the 5' overhangs. The resulting fragment was ligated with the Reading Frame A Gateway cassette (Invitrogen). To generate pENTR-PPARG2, the human PPARG2 cDNA was amplified from a publicly available plasmid (Addgene \#11439) and recombination sites compatible with the Gateway system (Invitrogen) were added. To generate lenti-PPARG2 plasmid, pENTRPPARG2 and pLV-tetO-attR1/R2 were recombined with LR Clonase II as described by the manufacturer's instructions (Invitrogen). All clones were confirmed by restriction digest screening and sequencing. Following a similar procedure, pENTR-eGFP, pENTR-PRDM16, pENTR-CEBPB, lenti-eGFP, lenti-PRDM16 and lenti-CEBPB were generated. The eGFP transcript was amplified from a commercially available plasmid (Addgene \#14430) as well as the PRDM16 (Origene NM_022114) and the CEBPB transcript (Origene NM_005194).

\section{Production of lentivirus and transduction}

A third-generation, Tat-free pack aging system ${ }^{49}$ was used to produce recombinant lentivirus. The vectors-either lenti-rtTA plasmid ${ }^{50-53}$ or lenti-PPARG2 plasmid-together with the two packaging plasmids-pMDL, pREV—and the plasmid coding for VSV-G envelope were transfected into HEK293T cells using calcium chlorate as previously described ${ }^{54}$ Cell media/viral supernatant was collected 48 and $72 \mathrm{~h}$ after transfection, passed through a $0.45 \mu \mathrm{m}$ filter and used directly for the transduction of cells. We determined the RNA copy numbers per millilitre of viral supernatant using the lenti-X RT-qPCR titration kit. The range varied between $7.5 \times 10^{7}$ copies $\mathrm{ml}^{-1}$ and $1.5 \times 10^{8}$ copies $\mathrm{ml}^{-1}$ and was influenced both by the packaged transgene and the specific preparation conditions.

Cells were transduced with lentiviral supernatant $24 \mathrm{~h}$ after passaging at about $40 \%$ confluency. Twelve-well dishes were transduced with $500 \mu \mathrm{l}$ lenti-rtTA virus and $500 \mu \mathrm{l}$ lenti-PPARG2 virus per well. Six-well dishes were transduced using $1000 \mu$ l lenti-rtTA virus and $1000 \mu 1$ lenti-PPARG2 virus per well. Cells were incubated at $37^{\circ} \mathrm{C}$ for $4 \mathrm{~h}$, viral supernatant was removed and cells were washed with PBS. Cells were further cultured in growth medium until differentiation was initiated through the administration of $700 \mathrm{ng} \mathrm{ml}^{-1}$ doxycycline and switch to adipogenic medium.

\section{Flow cytometry analysis}

Cells were trypsinized, washed with PBS and centrifuged at $200 g$ for 5 min. Cells were counted and $1 \times 10^{6}$ cells were transferred into polypropylene tubes. Staining for surface antigens was carried out using antibody conjugated to PE, FITC, PE-Cy7 or PE-Cy5 (PECD73 and ecto-5' nucleotidase AD2, BD Pharmingen; PE-CD105 (Endoglin) eBioscience; PE-Stro1, Santa Cruz; FITC-CD44, eBioscience; PE-Cy5-CD29, eBioscience; PE-Cy7CD4, BD Pharmingen; PE-CD19, eBioscience). Typically $1 \times 10^{6}$ cells in 80 or $95 \mu \mathrm{l}$ PBS with $5 \%$ FBS were stained using $20 \mu \mathrm{l}$ or $5 \mu \mathrm{l}$ antibody solution (final volume $100 \mu \mathrm{l}$ ). Tenthousand events per cell type were acquired on a FACSCalibur flow cytometer (BD Biosciences). Data were analysed using the FlowJo software package (Treestar).

\section{Immunocytochemistry}

Immunostaining was carried out using the following antibodies and dilutions: a-FABP4 1:200 (R\&D Systems), a-perilipin 1:200 (Sigma), a-CEBPA 1:100 (Santa Cruz), aPPARG2 1:200 (Santa Cruz), a-MAB1281 1:50 (Millipore), a-PRDM16 1:100 (Sigma), a- 
CEBPB 1:100 (Sigma), a-UCP1 1:200 (Sigma), MitoTracker (Invitrogen) and Alexa Fluor secondary antibodies (Invitrogen). Lipid droplets were stained using BODIPY neutral lipid dye. Hoechst or DAPI stain was used to mark cell nuclei. Images were acquired with either a Nikon Digital Sight camera mounted to a Nikon Eclipse Ti-S microscope, an Olympus DP72 camera mounted to a Olympus 1X71 microscope or a Zeiss LSM510 Meta confocal microscope. The NIS-Elements and Olympus DP2-BSW software packages were used for image analysis.

\section{RNA extraction, cDNA synthesis and RT-qPCR}

Total RNA from human cell lines and human fat was extracted with Trizol (Invitrogen) and purified using the RNeasy mini kit (Qiagen) according to the manufacturer's instructions. The RNA yield was determined using the NanoDrop ND-1000 spectrophotometer (NanoDrop Technologies). Total RNA $(1 \mu \mathrm{g})$ was converted to cDNA using the Superscript First-Strand kit (Invitrogen). RT-qPCR was carried out using a Realplex Mastercycler (Eppendorf) with the Quantifast-SYBR Green PCR mix (Qiagen) with $1 \mu$ cDNA per reaction. Primer sequences are listed in Supplementary Fig. S1D.

\section{Transcriptional profiling}

Total RNA from human cell lines or human fat was extracted with Trizol (Invitrogen) and purified using the RNeasy mini kit (Qiagen) according to the manufacturers' instructions. RNA quality was assessed using an Agilent bioanalyser. RNA probes for microarray hybridization were prepared and hybridized to the Affymetrix GeneChip Human Gene 1.0 ST microarray, Human Genome U133 Plus 2.0 array and Agilent GE G3 arrays.

Microarrays were quantile normalized and median centred using the RMA feature in the Bioconductor affy package in R. Probe sets were mapped to and median collapsed on to HUGO gene symbol identifiers. Agilent features were extracted using the Feature Extraction software. Normalization for the Agilent arrays was carried out by converting $\log _{2}$ intensity values to ratios of the 75th percentile intensity for each array and median centred. Agilent arrays were also batch corrected using the ComBat software package in R (ref. 55). SAM was used to rank order genes present on both arrays to calculate the Spearman rank correlation, which is the Pearson correlation of rank-ordered lists. The hypergeometric test to measure overlap between differentially expressed lists from both platforms was carried out in R. Unsupervised hierarchical clustering was carried out using Pearson's correlation as a similarity metric across all probes with Cluster 3.0. Heat maps were generated with Java TreeView. Differential expression was determined using the Samr package in $\mathrm{R}$ using a cutoff of 5\% local FDR. GO term analysis was carried out on differentially expressed genes using DAVID Bioinformatics Resources 6.7 (ref. 56) with the HuGene 1.0 ST microarray background.

All generated data sets have been submitted to publicly available databases under GEO accession numbers: GSE30041 SuperSeries, GSE30038 Affymetrix, GSE30039 Agilent.

\section{Lipolysis assay}

To measure lipolysis activity, day 21 adipocytes, differentiated from both hPSCs and ADSVCs, were starved in DMEM containing 1\% FBS for $1 \mathrm{~h}$. Cells were then incubated in HBSS (Hank's balanced salt solution) with 2\% fatty-acid-free BSA alone, with 1-10 $\mu \mathrm{M}$ isoproterenol or with $10 \mu \mathrm{M}$ forskolin. The culture medium was collected for glycerol measurement using the free glycerol reagent (Sigma \#F6428). Protein concentrations used to normalize glycerol content were measured using the Bradford protein assay (BioRad). Glycerol release was expressed in micrograms of glycerol per milligram of total protein or the lowest value is set to 1 . 


\section{Adiponectin and leptin ELISA}

Cell culture supernatants were analysed for adiponectin using the Protein Immunoassay kit (Millipore Corporation) as per the manufacturer's protocol. Briefly, the samples and kit controls were loaded onto a Millipore Multiscreen BV 96-well filter plate. Serial dilutions of standards were prepared in parallel and added to the plate. Millipore Multiplex beads were vortexed and sonicated for $30 \mathrm{~s}$ and $25 \mu \mathrm{l}$ was added to each well. Samples were incubated on a plate shaker at 600 r.p.m. in the dark at $4{ }^{\circ} \mathrm{C}$ overnight. The plate was applied to a Millipore multiscreen vacuum manifold and washed twice with $200 \mu 1$ of wash buffer. A 50 $\mu l$ volume containing biotinylated human adiponectin and leptin detection antibodies was added to each well. The plate was incubated on a plate shaker at 600 r.p.m. in the dark at room temperature for $1 \mathrm{~h}$. Streptavidin-phycoerythrin $(50 \mu \mathrm{l})$ was added directly to each well. The plate was incubated on a plate shaker at 600 r.p.m. in the dark at room temperature for $30 \mathrm{~min}$. The plate was applied to the vacuum manifold and washed twice with $200 \mu \mathrm{l}$ of wash buffer, and each well was resuspended in $100 \mu$ l sheath fluid and shaken for $5 \mathrm{~min}$. The assay plate was transferred to a Luminex 200 instrument for analysis. Cytokine concentrations were calculated using Upstate Beadview software with a five-parameter curve-fitting algorithm applied for standard-curve calculations.

\section{Metabolomic profiling}

All experiments were carried out using three biological replicates of cells grown on $10 \mathrm{~cm}$ plates. Medium was replaced $2 \mathrm{~h}$ before the extraction of the lipids. Ice-cold isopropanol (3 $\mathrm{ml}$; HPLC grade) was added, and cells were scraped and transferred into $15 \mathrm{ml}$ centrifuge tubes. The extracts were incubated for $1 \mathrm{~h}$ at $4{ }^{\circ} \mathrm{C}$ and then vortexed and centrifuged at $2,300 \mathrm{~g}$. for $10 \mathrm{~min}$. The supernatant was transferred to a new $15 \mathrm{ml}$ centrifuge tube and stored at $-20^{\circ} \mathrm{C}$.

All data were acquired using an Applied Biosystems QSTAR XL hybrid quadrupole/timeof-flight mass spectrometer ${ }^{57}$. MultiQuant software (version 1.1; Applied Biosystems/Sciex) was used for automated peak integration, and peaks were manually reviewed for quality of integration. Internal standard peak areas were monitored for quality control and used to normalize analyte peak areas.

\section{Western blot analysis}

BJ-RiPS-MPC-derived adipocytes were starved overnight in DMEM containing 2\% fatty acid free BSA. To induce insulin resistance, FFA mixture (lauric, myristic, linolein, oleic and arachidonic acids from Sigma) was used at $0.5 \mathrm{mM}-1 \mathrm{mM}$ for $1 \mathrm{~h}$. Insulin stimulation was carried out at $100 \mathrm{nM}$ for $20 \mathrm{~min}$. Cells were lysed in cold RIPA buffer (60 mM Tris$\mathrm{HCl}$ at $\mathrm{pH} 7.4,150 \mathrm{mM} \mathrm{NaCl}, 0.25 \% \mathrm{SDS}$ and $1 \% \mathrm{NP} 40$ ) containing $10 \mathrm{mM} \mathrm{NaF}, 1 \mathrm{mM}$ $\mathrm{Na}_{3} \mathrm{VO}_{4}$ and complete protease inhibitor (Roche). Western blot analysis was carried out using the following antibodies: total AKT (Cell Signaling \#9272), phospho-Ser-473 AKT (Cell Signaling \#9271) and lamin A/C (Fisher/Millipore \#3211).

\section{Glucose uptake assay}

Adipocytes were serum-starved in $0.2 \%$ BSA DMEM overnight. Then, cells were incubated in KRH buffer $\left(121 \mathrm{mM} \mathrm{NaCl}, 4.9 \mathrm{mM} \mathrm{KCl}, 1.2 \mathrm{mM} \mathrm{MgSO}_{4}, 0.33 \mathrm{mM} \mathrm{CaCl}_{2}\right.$ and $12 \mathrm{mM}$ HEPES, at $\mathrm{pH} 7.4$ ) in the absence or presence of $100 \mathrm{nM}$ insulin for $30 \mathrm{~min}$ at $37^{\circ} \mathrm{C}$, followed by washing three times in KRH buffer. Glucose uptake was measured by incubating cells with $0.5 \mu \mathrm{Ci} \mathrm{ml}^{-1}$ 2-deoxy-d- $\left[{ }^{3} \mathrm{H}\right]$ glucose (Perkin-Elmer) for $5 \mathrm{~min}$ at 37 ${ }^{\circ} \mathrm{C}$. After three washes with cold PBS, cells were lysed with $1 \%$ Triton X-100 solution and subjected to scintillation counting. Nonspecific uptake was measured in the presence of 10 $\mu \mathrm{M}$ cytochalasin B and was subtracted from the total uptake. 


\section{Transplantation}

PPARG2- or PPARG2-CEBPB-transduced HUES 9 MPCs were differentiated for 14 days. To avoid rejection, differentiated cells were injected subcutaneously into $\mathrm{Rag}^{-/-}$; IL $\gamma \mathrm{c}$ mice. Four-six weeks after transplantation, mice were killed to collect fat pads. 3T3-F442A cells were injected into the same mice as a positive control. Tissues at the transplantation site were embedded in paraffin and stained after sectioning.

\section{PET analysis}

To detect the molecular activity of programmed human white and brown adipose tissue, mice were intravenously injected with $\left[{ }^{18} \mathrm{~F}\right] \mathrm{FDG}$, a glucose analogue, $2 \mathrm{~h}$ before PET acquisition. The skin with transplanted human adipose tissue was removed and scanned to visualize the PET signal in the ex vivo sample. PET imaging was carried out over $2 \mathrm{~h}$ using an Inveon small-animal scanner (Siemens). Osirix was used for visualization of the DICOM images and obtained images were reconstructed using IRM software.

\section{Measurement of cellular OCR and ECAR}

Cells were plated in gelatin-coated XF 24-well cell culture microplates (Seahorse Bioscience) and differentiated into adipocytes. Cells were incubated in pre-warmed unbuffered DMEM medium (DMEM containing $2 \mathrm{mM}$ GlutaMAX, $1 \mathrm{mM}$ sodium pyruvate, $1.85 \mathrm{~g} \mathrm{l}^{-1} \mathrm{NaCl}$ and $25 \mathrm{mM}$ glucose) for $1 \mathrm{~h}$. The oxygen consumption was measured by the XF24 extracellular flux analyser (Seahorse Biosciences). Mitochondrial biogenesis was profiled by injecting perturbation drugs, $2 \mu \mathrm{M}$ oligomycin, $0.5 \mu \mathrm{MFCCP}$ (carbonyl cyanide$p$-trifluoromethoxyphenylhydrazone) and $5 \mu \mathrm{M}$ antimycin $\mathrm{A}$, in succession. OCR and ECAR were determined by plotting the oxygen tension and acidification of the medium in the chamber as a function of time and normalized by protein concentration (picomoles per minute per milligram), respectively.

\section{Supplementary Material}

Refer to Web version on PubMed Central for supplementary material.

\section{Acknowledgments}

The authors would like to thank T. Holm, A. Foudi and E. Hanson for critical reading, insight and suggestions; M. Henderson, J. Hom, K. Hom, I. Pomerantseva, A. Tseng and K. Kulig for their technical help; L. Prickett-Rice and K. Folz-Donahue of the HSCI-CRM Flow Cytometry Core Facility; D. Lieber and V. Mootha for assistance with cellular bioenergetic measurements; and J. Truelove and R. Weissleder for assistance with PET-CT imaging. T. Ahfeldt was supported by the Roberto and Allison Mignone Fund for Stem Cell Research. This work was financially sponsored in part by the Harvard Stem Cell Institute, Hoffman-La Roche and the Stowers Medical Institute.

\section{References}

1. Thomson JA, et al. Embryonic stem cell lines derived from human blastocysts. Science. 1998; 282:1145-1147. [PubMed: 9804556]

2. Takahashi K, et al. Induction of pluripotent stem cells from adult human fibroblasts by defined factors. Cell. 2007; 131:861-872. [PubMed: 18035408]

3. Yu J, et al. Induced pluripotent stem cell lines derived from human somatic cells. Science. 2007; 318:1917-1920. [PubMed: 18029452]

4. Park IH, et al. Disease-specific induced pluripotent stem cells. Cell. 2008; 134:877-886. [PubMed: 18691744]

5. Lee G, et al. Modelling pathogenesis and treatment of familial dysautonomia using patient-specific iPSCs. Nature. 2009; 461:402-406. [PubMed: 19693009] 
6. Green H, Kehinde O. An established preadipose cell line and its differentiation in culture. II. Factors affecting the adipose conversion. Cell. 1975; 5:19-27. [PubMed: 165899]

7. Farmer SR. Transcriptional control of adipocyte formation. Cell Metab. 2006; 4:263-273. [PubMed: 17011499]

8. Tontonoz P, Hu E, Graves RA, Budavari AI, Spiegelman BM. mPPAR 2: tissue-specific regulator of an adipocyte enhancer. Genes Dev. 1994; 8:1224-1234. [PubMed: 7926726]

9. Tontonoz P, Hu E, Spiegelman BM. Stimulation of adipogenesis in fibroblasts by PPAR 2, a lipidactivated transcription factor. Cell. 1994; 79:1147-1156. [PubMed: 8001151]

10. Cannon B, Nedergaard J. Brown adipose tissue: function and physiological significance. Physiol Rev. 2004; 84:277-359. [PubMed: 14715917]

11. Cypess AM, et al. Identification and importance of brown adipose tissue in adult humans. New Engl J Med. 2009; 360:1509-1517. [PubMed: 19357406]

12. Saito M, et al. High incidence of metabolically active brown adipose tissue in healthy adult humans: effects of cold exposure and adiposity. Diabetes. 2009; 58:1526-1531. [PubMed: 19401428]

13. Virtanen KA, et al. Functional brown adipose tissue in healthy adults. New Engl J Med. 2009; 360:1518-1525. [PubMed: 19357407]

14. Seale P, et al. Transcriptional control of brown fat determination by PRDM16. Cell Metab. 2007; 6:38-54. [PubMed: 17618855]

15. Kajimura $S$, et al. Initiation of myoblast to brown fat switch by a PRDM16-C/EBP- $\beta$ transcriptional complex. Nature. 2009; 460:1154-1158. [PubMed: 19641492]

16. Pittenger MF, et al. Multilineage potential of adult human mesenchymal stem cells. Science. 1999; 284:143-147. [PubMed: 10102814]

17. Lazarus HM, Haynesworth SE, Gerson SL, Rosenthal NS, Caplan AI. Ex vivo expansion and subsequent infusion of human bone marrow-derived stromal progenitor cells (mesenchymal progenitor cells): implications for therapeutic use. Bone Marrow Transplant. 1995; 16:557-564. [PubMed: 8528172]

18. Zuk PA, et al. Multilineage cells from human adipose tissue: implications for cell-based therapies. Tissue Eng. 2001; 7:211-228. [PubMed: 11304456]

19. Barberi T, Studer L. Mesenchymal cells. Methods Enzymol. 2006; 418:194-208. [PubMed: 17141037]

20. Dani C. Embryonic stem cell-derived adipogenesis. Cells Tissues Organs. 1999; 165:173-180. [PubMed: 10592389]

21. Xiong C, et al. Derivation of adipocytes from human embryonic stem cells. Stem Cells Dev. 2005; 14:671-675. [PubMed: 16433622]

22. van Harmelen V, et al. Differential lipolytic regulation in human embryonic stem cell-derived adipocytes. Obesity (Silver Spring). 2007; 15:846-852. [PubMed: 17426319]

23. Hannan NR, Wolvetang EJ. Adipocyte differentiation in human embryonic stem cells transduced with Oct4 shRNA lentivirus. Stem Cells Dev. 2008; 18:653-660. [PubMed: 18764734]

24. Barberi T, Willis LM, Socci ND, Studer L. Derivation of multipotent mesenchymal precursors from human embryonic stem cells. PLoS Med. 2005; 2:e161. [PubMed: 15971941]

25. Trivedi P, Hematti P. Derivation and immunological characterization of mesenchymal stromal cells from human embryonic stem cells. Exp Hematol. 2008; 36:350-359. [PubMed: 18179856]

26. Warren L, et al. Highly efficient reprogramming to pluripotency and directed differentiation of human cells with synthetic modified mRNA. Cell Stem Cell. 2010; 7:618-630. [PubMed: 20888316]

27. Olivier EN, Rybicki AC, Bouhassira EE. Differentiation of human embryonic stem cells into bipotent mesenchymal stem cells. Stem Cells. 2006; 24:1914-1922. [PubMed: 16644919]

28. Sen A, et al. Adipogenic potential of human adipose derived stromal cells from multiple donors is heterogeneous. J Cell Biochem. 2001:312-319. [PubMed: 11241671]

29. Tashiro K, et al. Efficient adenovirus vector-mediated PPAR $\gamma$ gene transfer into mouse embryoid bodies promotes adipocyte differentiation. J Gene Med. 2008; 10:498-507. [PubMed: 18278825] 
30. Bermingham JR Jr, et al. Identification of genes that are downregulated in the absence of the POU domain transcription factor pou3f1 (Oct-6, Tst-1, SCIP) in sciatic nerve. J Neurosci. 2002; 22:10217-10231. [PubMed: 12451123]

31. Rosen ED, et al. PPAR $\gamma$ is required for the differentiation of adipose tissue in vivo and in vitro. Mol Cell. 1999; 4:611-617. [PubMed: 10549292]

32. Maherali N, et al. A high-efficiency system for the generation and study of human induced pluripotent stem cells. Cell Stem Cell. 2008; 3:340-345. [PubMed: 18786420]

33. Seale P, et al. PRDM16 controls a brown fat/skeletal muscle switch. Nature. 2008; 454:961-967. [PubMed: 18719582]

34. Altiok S, Xu M, Spiegelman BM. PPAR $\gamma$ induces cell cycle withdrawal: inhibition of E2F/DP DNA-binding activity via down-regulation of PP2A. Genes Dev. 1997; 11:1987-1998. [PubMed: 9271121]

35. Rosen ED, MacDougald OA. Adipocyte differentiation from the inside out. Nat Rev Mol Cell Biol. 2006; 7:885-896. [PubMed: 17139329]

36. Svensson PA, et al. Gene expression in human brown adipose tissue. Int J Mol Med. 2011; 27:227232. [PubMed: 21125211]

37. Tusher VG, Tibshirani R, Chu G. Significance analysis of microarrays applied to the ionizing radiation response. Proc Natl Acad Sci USA. 2001; 98:5116-5121. [PubMed: 11309499]

38. Huang da W, Sherman BT, Lempicki RA. Systematic and integrative analysis of large gene lists using DAVID bioinformatics resources. Nat Protoc. 2009; 4:44-57. [PubMed: 19131956]

39. Huang da W, Sherman BT, Lempicki RA. Bioinformatics enrichment tools: paths toward the comprehensive functional analysis of large gene lists. Nucleic Acids Res. 2009; 37:1-13. [PubMed: 19033363]

40. Puigserver $P$, et al. A cold-inducible coactivator of nuclear receptors linked to adaptive thermogenesis. Cell. 1998; 92:829-839. [PubMed: 9529258]

41. de Jesus LA, et al. The type 2 iodothyronine deiodinase is essential for adaptive thermogenesis in brown adipose tissue. J Clin Invest. 2001; 108:1379-1385. [PubMed: 11696583]

42. Zhang Y, et al. Positional cloning of the mouse obese gene and its human homologue. Nature. 1994; 372:425-432. [PubMed: 7984236]

43. Simpson F, Whitehead JP. Adiponectin-it's all about the modifications. Int J Biochem Cell Biol. 2010; 42:785-788. [PubMed: 20044026]

44. Rhee EP, et al. Metabolite profiling identifies markers of uremia. J Am Soc Nephrol. 2010; 21:1041-1051. [PubMed: 20378825]

45. Jiao P, et al. FFA-induced adipocyte inflammation and insulin resistance: involvement of ER stress and IKK $\beta$ pathways. Obesity (Silver Spring). 2011; 19:483-491. [PubMed: 20829802]

46. Nedergaard J, Bengtsson T, Cannon B. Unexpected evidence for active brown adipose tissue in adult humans. Am J Physiol Endocrinol Metab. 2007; 293:E444-E452. [PubMed: 17473055]

47. van Marken Lichtenbelt WD, et al. Cold-activated brown adipose tissue in healthy men. New Engl J Med. 2009; 360:1500-1508. [PubMed: 19357405]

48. Stadtfeld M, Brennand K, Hochedlinger K. Reprogramming of pancreatic beta cells into induced pluripotent stem cells. Curr Biol. 2008; 18:890-894. [PubMed: 18501604]

49. Tiscornia G, Singer O, Verma IM. Production and purification of lentiviral vectors. Nat Protoc. 2006; 1:241-245. [PubMed: 17406239]

50. Maherali N, et al. A high-efficiency system for the generation and study of human induced pluripotent stem cells. Cell Stem Cell. 2008; 3:340-345. [PubMed: 18786420]

51. Maherali N, et al. Directly reprogrammed fibroblasts show global epigenetic remodeling and widespread tissue contribution. Cell Stem Cell. 2007; 1:55-70. [PubMed: 18371336]

52. Park IH, et al. Disease-specific induced pluripotent stem cells. Cell. 2008; 134:877-886. [PubMed: 18691744]

53. Stadtfeld M, Maherali N, Breault DT, Hochedlinger K. Defining molecular cornerstones during fibroblast to iPS cell reprogramming in mouse. Cell Stem Cell. 2008; 2:230-240. [PubMed: 18371448] 
54. al Yacoub N, Romanowska M, Haritonova N, Foerster J. Optimized production and concentration of lentiviral vectors containing large inserts. J Gene Med. 2007; 9:579-584. [PubMed: 17533614]

55. Johnson WE, Li C, Rabinovic A. Adjusting batch effects in microarray expression data using empirical Bayes methods. Biostatistics. 2007; 8:118-127. [PubMed: 16632515]

56. Huang da W, Sherman BT, Lempicki RA. Systematic and integrative analysis of large gene lists using DAVID bioinformatics resources. Nat Protoc. 2009; 4:44-57. [PubMed: 19131956]

57. Rhee EP, et al. Metabolite profiling identifies markers of uremia. J Am Soc Nephrol. 2010; 21:1041-1051. [PubMed: 20378825] 


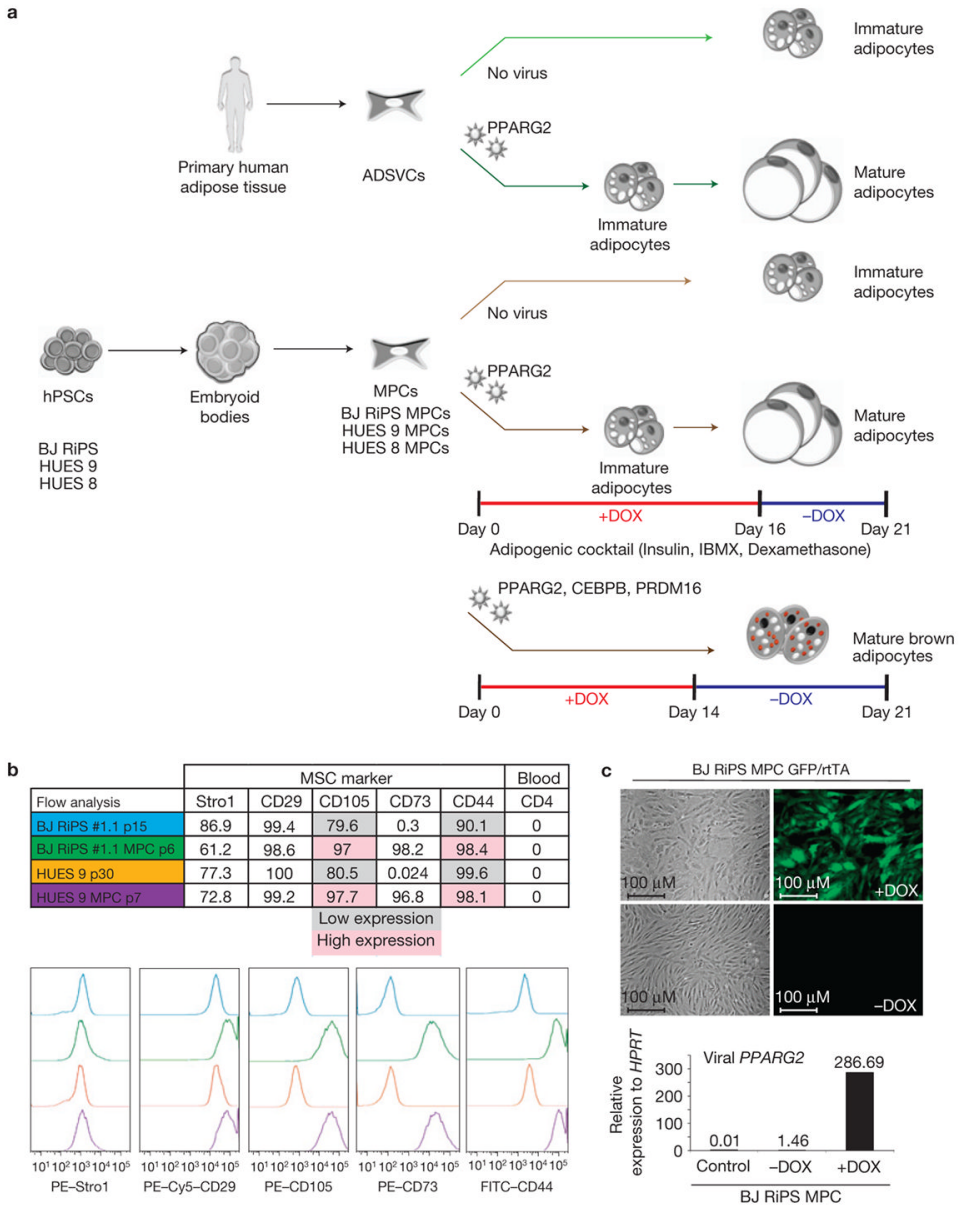

Figure 1.

Experimental scheme and characterization of hPSC-derived MPCs. (a) Experimental scheme for the differentiation of ADSVCs and hPSCs into white and brown adipocytes. hPSCs were differentiated as embryoid bodies and then re-plated and passaged to generate MPCs. MPCs and ADSVCs were either not infected (unprogrammed) or transduced (programmed) with lentivirus constitutively expressing the lenti-rtTA M2 domain and virus carrying the inducible cDNA transgenes PPARG2 (lenti-PPARG2), CEBPB (lenti-CEBPB) and PRDM16 (lenti-PRDM16). All differentiated cells were cultured in media containing adipogenic factors and doxycycline for 14-16 days and maintained without doxycycline until 21 days before analysis. Four hPSC lines were used-two hESC lines, HUES 8 and 9, and two induced pluripotent stem cell (iPSC) clones generated by reprogramming BJ fibroblasts with modified ribonucleic acids, BJ RiPSC. (b) Top, table showing the results of the flow cytometry analysis of the hPSC lines BJ RiPS \#1.1 and HUES 9 as well as MPCs derived from these lines. Cells were stained for the surface antigens Stro1, CD105, CD73, CD44, CD29 and CD4. The numbers represent the percentage of positive cells. Where appropriate, positive stainings were distinguished as high- or low-expression groups. Bottom, flow cytometry results for the surface antigens Stro1, CD105, CD73, CD44 and CD29 presented as histograms: BJ RiPS \#1.1 p15 (blue), BJ RiPS \#1.1 MPC p6 (green), HUES 9 p30 (orange) and HUES 9 MPC p7 (violet). The $x$ axis indicates the relative fluorescence intensity from 10 to 100.000 on a logarithmic scale. The $y$ axis represents the percentage of cells. (c) Doxycycline-inducible expression of PPARG2 and eGFP. Top, BJ RiPS MPCs transduced with lenti-rtTA and doxycycline-inducible eGFP virus and cultured 
either in the presence (+DOX; top panels) or absence (-DOX; bottom); Bottom, RT-qPCR assays for viral PPARG2 cDNA expression normalized to HPRT: BJ RiPS MPCs

transduced with lenti-rtTA only (control); BJ RiPS MPCs transduced with lenti-rtTA and lenti-PP ARG2 cultured in the absence (-DOX) or presence (+DOX) of doxycycline. 


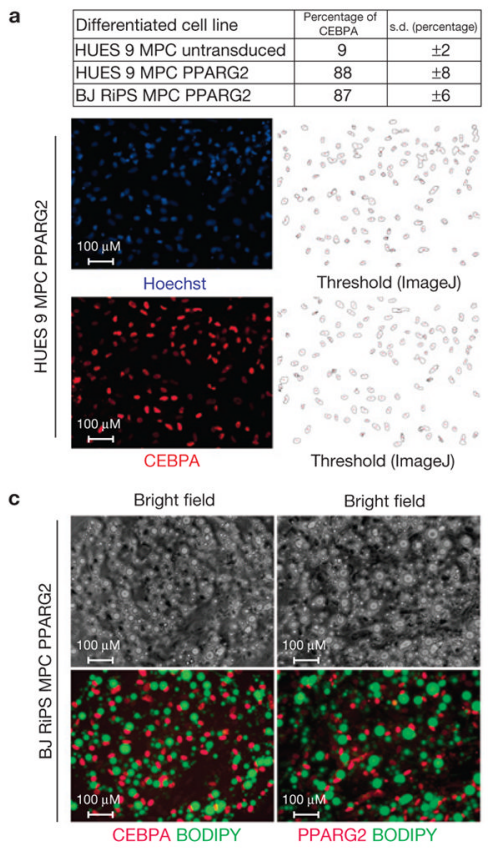

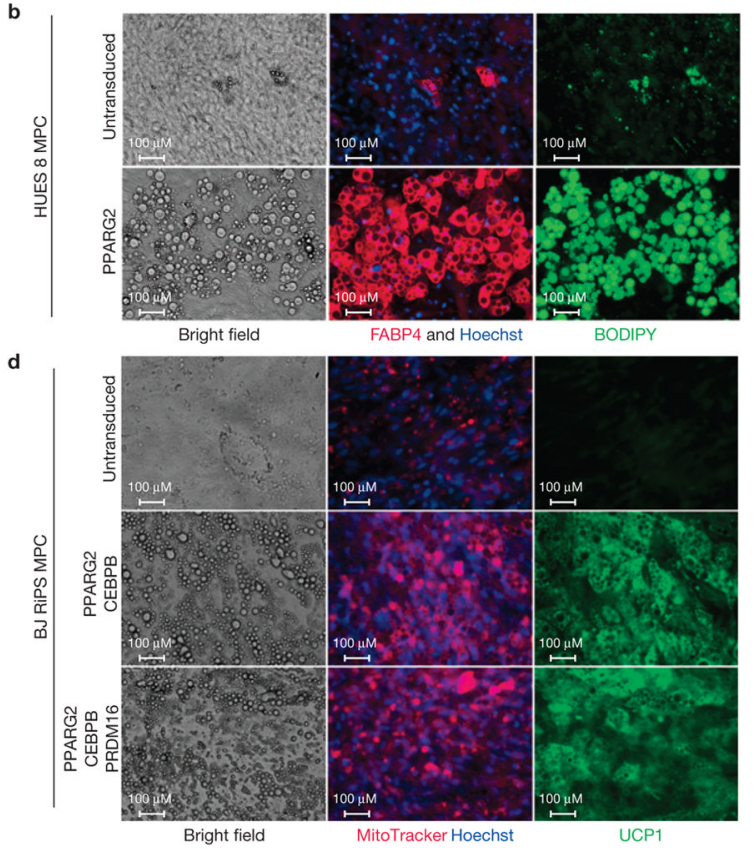

Figure 2.

Programming hPSC-derived MPCs with PPARG2, PPARG2-CEBPB or PPARG2CEBPB-PRDM16 generates white and brown adipocytes. (a) Efficiency of white adipocyte differentiation. Top, table showing the efficiency of white adipocyte formation from cells in adipogenic media alone (untransduced) or PPARG2-programmed differentiated cells (PPARG2) as determined by the ratio of Hoechst-positive and CEBPA-positive nuclei. Bottom, representative images of Hoechst-stained (top left panel) and CEBPA-stained (bottom left panel) nuclei from HUES 9 MPCs programmed with PPARG2 $(\times 100$ magnification). The right panels illustrate the threshold assignment of positive nuclei and counting carried out by the ImageJ analysis software. (b) Programming hPSCs into white adipocytes. HUES-8-derived MPCs were differentiated with adipogenic media alone (top panels; untransduced) or in combination with exogenous PPARG2 expression (bottom panels; PPARG2). From left to right: bright-field images illustrating the morphology of immature (top panel) and mature (lower panel) white adipocytes; fluorescence micrographs of corresponding immunostains with antibodies against the adipocyte marker protein FABP4 (red) and the neutral lipid dye BODIPY (green); all cells were co-stained with Hoechst (blue) to identify nuclei ( $\times 100$ magnification). (c) hPSC-derived white adipocytes express endogenous CEBPA and PPARG2. BJ-RiPSC-derived MPCs were differentiated and programmed with exogenous $P P A R G 2$ expression for 16 days and, 5 days after withdrawal of doxycycline, stained with antibodies against CEBPA (red; lower left panel) or PPARG2 (red; lower right panel). All cells were also stained with the neutral lipid dye BODIPY (green, both lower panels, $\times 100$ magnification). The upper panels show corresponding bright-field images. (d) hPSC-derived brown adipocytes express UCP1 and can be efficiently labelled with MitoTracker. BJ-RiPSC-derived MPCs were differentiated with adipogenic media alone (top panels; untransduced) or programmed with either exogenous $P P A R G 2+C E B P B$ (middle panel) or exogenous PPARG2 + CEBPB + PRDM16 (lower panel) expression for 14 days and, 7 days after withdrawal of doxycycline, labelled with MitoTracker (red) or stained with antibodies against UCP1 (green). The left panels show corresponding bright-field images (all images $\times 200$ magnification). 

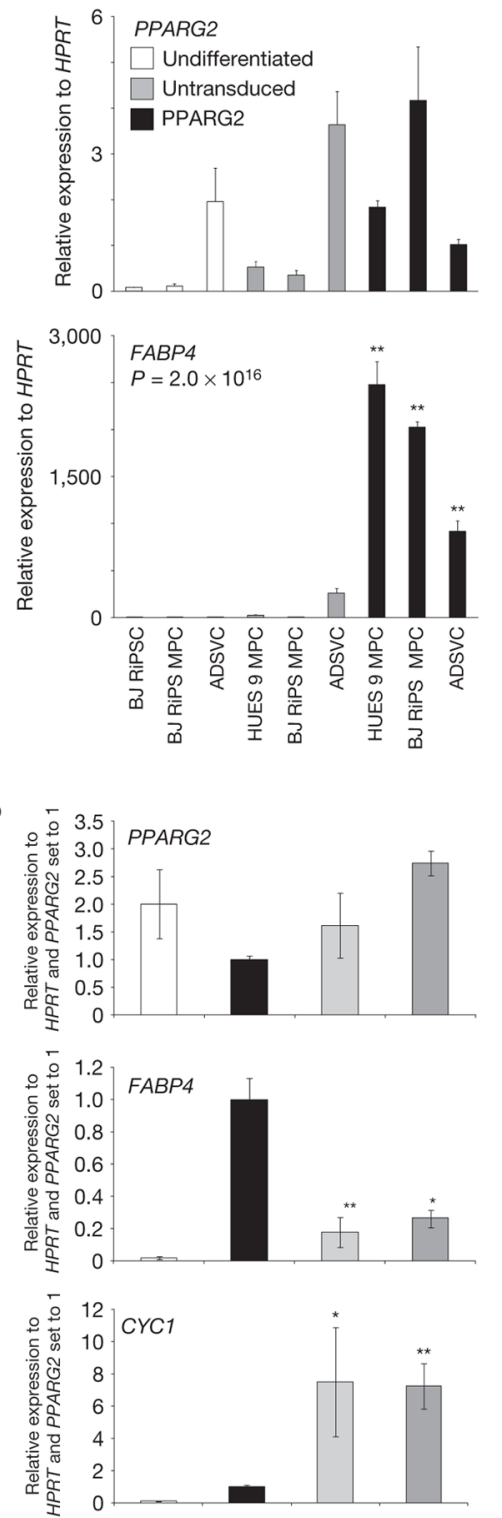

$\square$ Untransduced
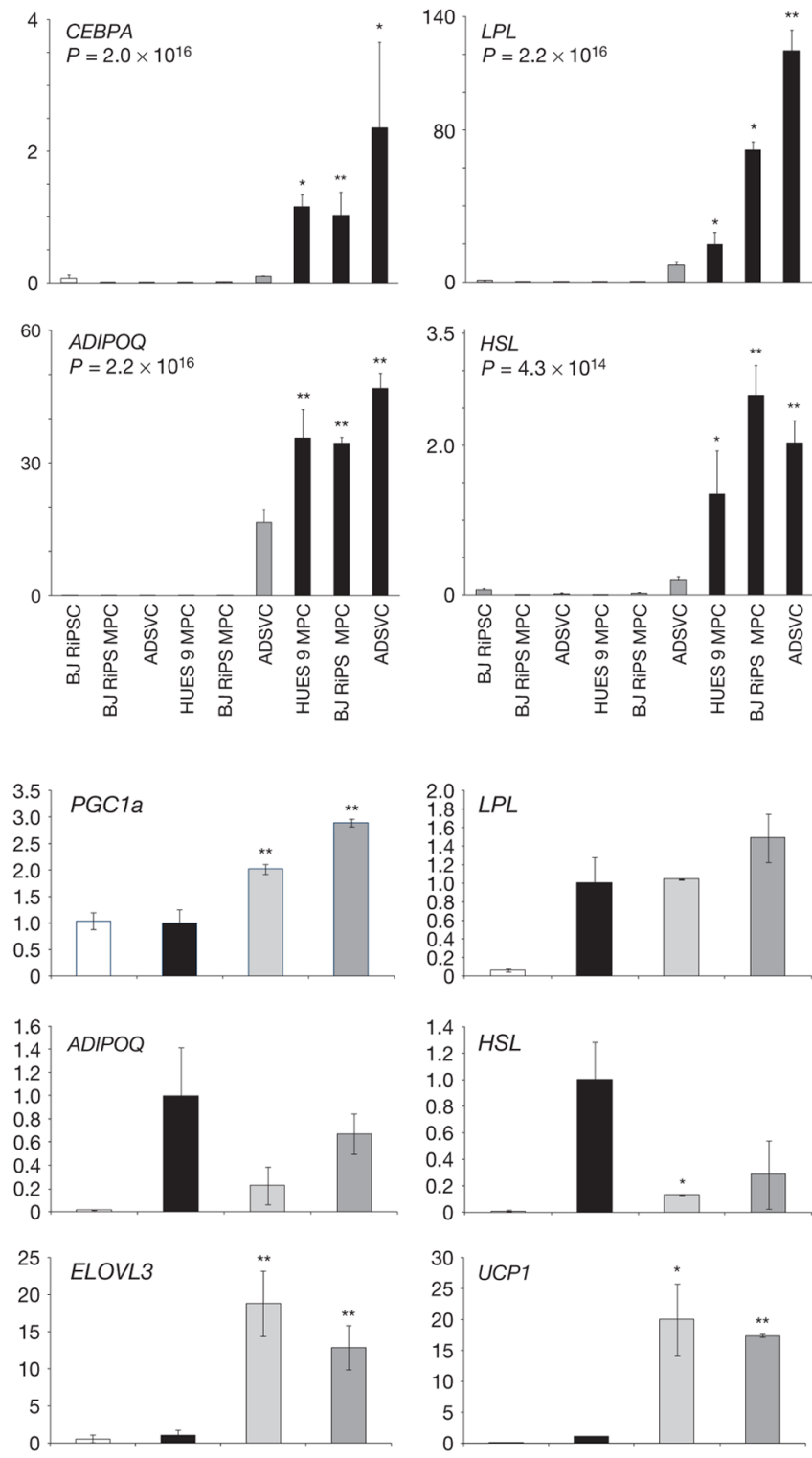

BJ RiPS \#1.1 p10 MPC p6

PPARG2 $\square$ PPARG2-CEBPB $\square$ PPARG2-CEBPB-PRDM16

Figure 3.

Programmed adipocytes exhibit a mature white or brown adipocyte gene expression profile. (a) hPSC-derived white adipocytes express mature marker genes. RT-qPCR assays were carried out for adipocyte marker genes PPARG2, CEBPA, FABP4, ADIPOQ, HSL and $L P L$. The expression values represent three biological replicates and are shown relative to $H P R T$ expression in each sample. White bars represent cells that were not exposed to adipogenic media (undifferentiated); grey bars represent cells that were exposed to adipogenic media but not transduced with lenti-PPARG2 (-PPARG2); black bars represent cells that were exposed to adipogenic media and transduced with lenti-PPARG2 (+PPARG2). $P$ values represent two-tailed Student's $t$-tests between - PPARG2 and + PPARG2 expression values for each cell line. $n=3, * P<0.05$, ${ }^{* *} P<0.01$ (s.d.). $P$ values shown under each gene name represent results of analysis of variance among all expression values for-PPARG2 and +PPARG2 cell lines. (b) Comparison of hPSC-derived white 
adipocytes and brown adipocytes. RT-qPCR assays were carried out for a range of white or brown adipocyte marker genes, PPARG2, PGC1a, FABP4, ADIPOQ, HSL, LPL, CYC1, $E L O V L 3$ and $U C P 1$. Expression values represent three biological replicates and are shown relative to HPRT expression and relative to the lenti-PPARG2 condition set as 1 . White bars represent cells that were differentiated with adipogenic media alone (untransduced); black bars represent cells that were exposed to adipogenic media and transduced with lentiPPARG2 (+PPARG2); grey bars represent cells that were exposed to adipogenic media and transduced with lenti-PPARG2 and lenti-CEBPB (+PPARG2-CEBPB, light grey bars) or with lenti-PPARG2, lenti-CEBPB and lenti-PRDM16 (+PPARG2-CEBPB-PRDM16, dark grey bars) respectively. All experiments were carried out with BJ-RiPSC-derived MPCs. $P$ values represent two-tailed Student's $t$-tests between the PPARG2 and PPARG2-CEBPB or PPARG2-CEBPB-PRDM16 set-ups respectively. Values for each cell line, $n=3$, ${ }^{*} P<$ $0.05, * * P<0.01$ (s.d.). 

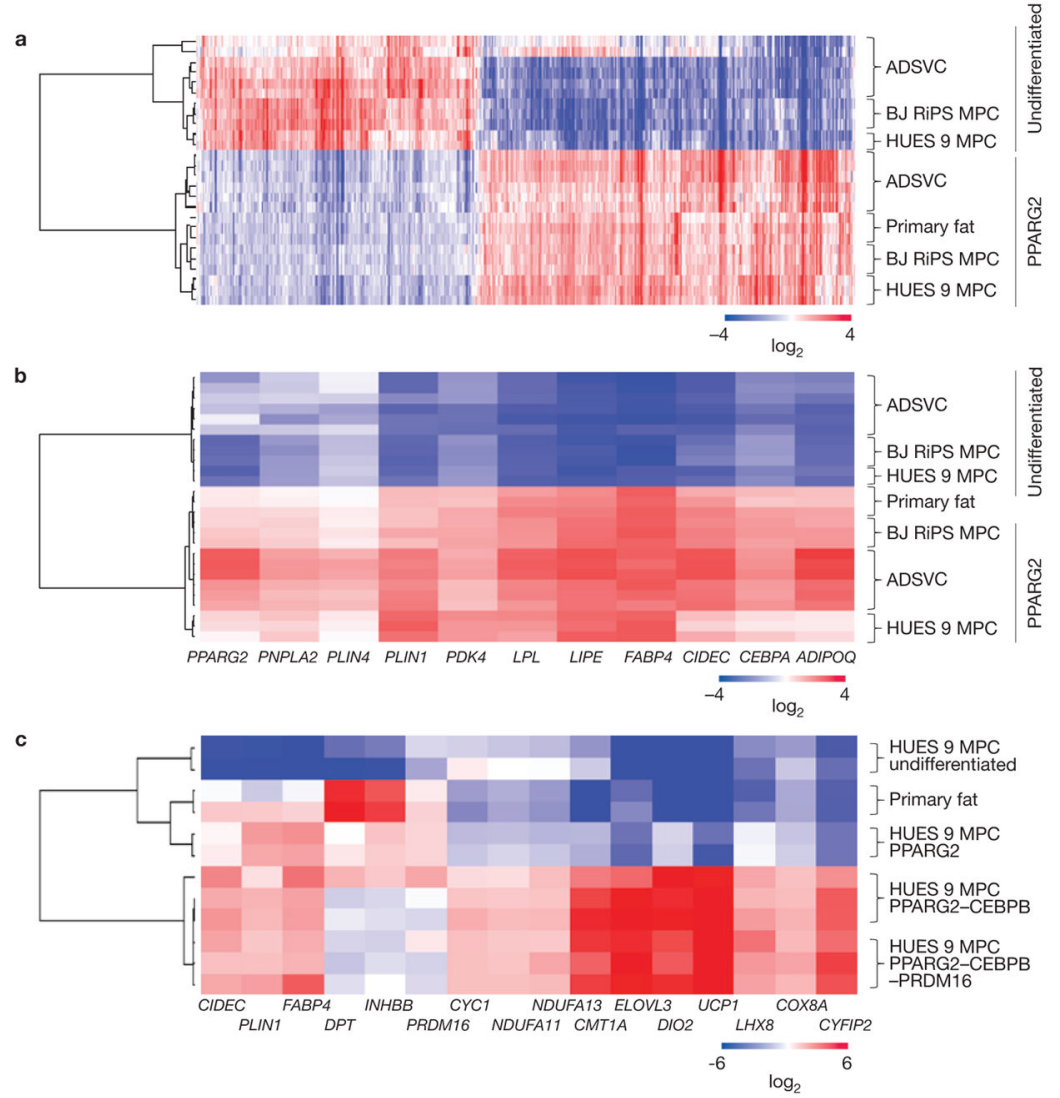

Figure 4.

Global transcriptional analysis confirms the identity of programmed hPSC-derived white and brown adipocytes. (a) ADSVCs, HUES 9 MPCs and BJ RiPS MPCs either not exposed to adipogenic media (undifferentiated) or cultured with adipogenic media and transduced with lenti-PPARG2 (+PPARG2) were compared with primary adipocytes using Affymetix 1.0 ST microarrays. Shown is a row-centred heat map of hierarchical clustering carried out on the 2,136 differentially expressed genes at a 5\% FDR. Probe sets are coloured according to the average expression level across all samples, with blue denoting a lower expression level and red denoting a higher expression level. (b) Row-centred heat map and hierarchical cluster of an adipo-specific gene panel across the same samples as in $\mathbf{a}$. Probe sets are coloured according to the average expression level across all samples, with blue denoting a lower expression level and red denoting a higher expression level. (c) Row-centred heat map hierarchical cluster of a brown and white adipo-specific gene panel across the same samples as in $\mathbf{a}$ and $\mathbf{b}$ (white adipocytes, primary white adipose tissue and undifferentiated MPCs) in addition to PPARG2-CEBPB-PRDM16-programmed MPCs (brown adipocytes) on the Agilent G3 human GE array platform. Probe sets are coloured according to the average expression level across all samples, with blue denoting a lower expression level and red denoting a higher expression level. 


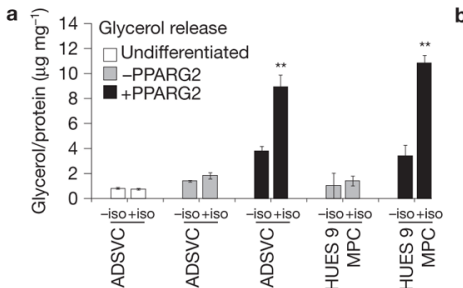

b
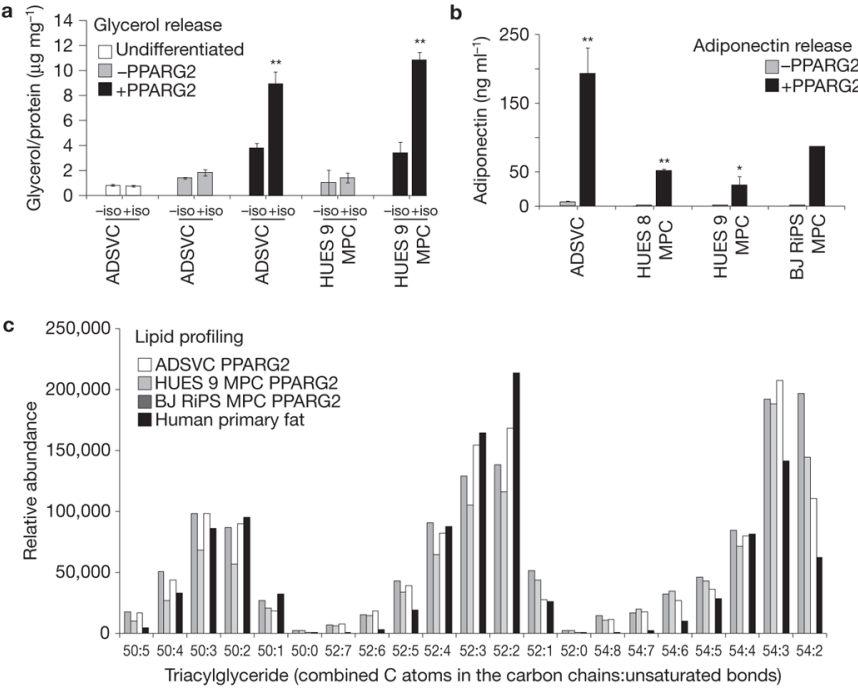

e

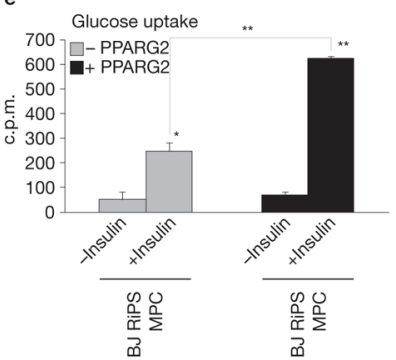

Figure 5.

Programmed hPSC-derived white adipocytes exhibit mature functional properties. (a) hPSCderived adipocytes carry out lipolysis. Glycerol was measured in the supernatant of ADSVCs and HUES-9-derived MPCs that were either not exposed to adipogenic media (undifferentiated) or exposed to adipogenic media without (-PPARG2) or with exogenous PPARG2 expression (+PPARG2) followed by either treatment with (+iso) or without (-iso) isoproterenol. The quantity of glycerol released (in micrograms) was normalized to the total amount of protein (in milligrams) for each sample. $n=3$, $* * P<0.01$ (s.e.m.). (b) hPSCderived adipocytes secrete adiponectin. ELISA for adiponectin in the supernatant of cells exposed to adipogenic media and either not transduced with lenti-PPARG2 (-PPARG2) or transduced with lenti-PPARG2 (+PPARG2). Experiments carried out as biological triplicates, with the exception of BJ RiPS MPCs. $n=3$, ${ }^{*} P<0.05$, $* * P<0.01$ (s.d.). (c) Lipid profiling of ADSVC- and hPSC-derived adipocytes. The cellular lipid content of PPARG2-programmed ADSVCs, HUES 9-derived MPCs and BJ-RiPS-derived MPCs was analysed using a tandem mass spectroscopy lipidomics platform and compared with the lipid content of primary adipose tissue. Shown are the relative abundances of several long-chain triacylglyceride species in each cell type. The $x$ axis denotes the total number of carbon atoms in the fatty-acid chains:unsaturated bonds. The $y$ axis represents the relative abundance of each lipid analyte. (d) Attenuation of insulin-induced Ser-473 phosphorylation on AKT by FFAs. BJ-RiPS-MPC-derived adipocytes were treated using either insulin alone, BSA-bound FFAs or with both. Phosphorylation of AKT was determined in the whole-cell lysate by immunoblotting with the phospho-specific AKT (Ser 473) antibody. (e) Glucose uptake in BJ-RiPS-MPC-derived adipocytes was assessed by the transport of $\left[{ }^{3} \mathrm{H}\right]$-2-deoxyD-glucose following insulin stimulation. MPCs were exposed to adipogenic media without (-PPARG2) or with exogenous PPARG2 expression (+PPARG2) followed by either treatment with (+insulin) or without (-insulin) insulin. The quantity of $\left[{ }^{3} \mathrm{H}\right] 2$-deoxy-Dglucose transported into the cells was normalized to CytoB and the results are shown as c.p.m. $n=3, * P<0.05, * * P<0.01$ (s.e.m.). Uncropped images of blots are shown in Supplementary Fig. S9. 


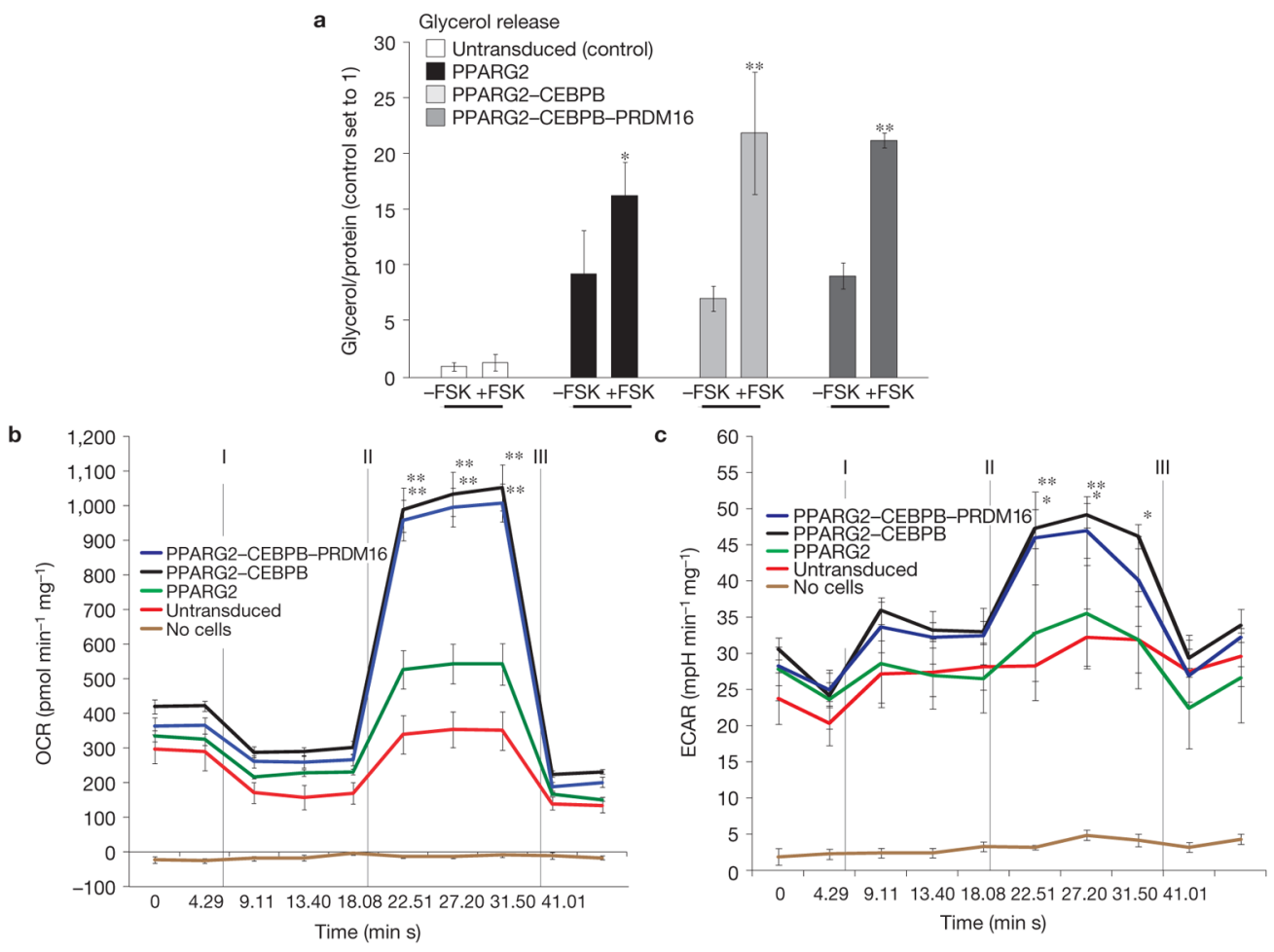

Figure 6.

Programmed hPSC-derived brown adipocytes demonstrate mature functional properties. (a) Glycerol release assay with hPSC-derived brown and white adipocytes. Glycerol was measured in the supernatant of HUES-9-derived MPCs that were differentiated with adipogenic media alone (untransduced, white bars), with exogenous PPARG2 expression (+PPARG2, black), with expression of a combination of lenti-PPARG2 and lenti-CEBPB (+PPARG2-CEBPB, light grey bars) or with a combination of lenti-PPARG2, lenti-CEBPB and lenti-PRDM16 (+PPARG2-CEBPB-PRDM16, dark grey bars). After differentiation of the cells, measurements were made at the basal level and after exposure to forskolin (+FSK). The quantity of released glycerol (in micrograms) was normalized to the total amount of protein (in milligrams) for each sample. $n=3$, Student's $t$-test $* * P<0.01$ (s.e.m.). (b,c) Comparison of the OCR and ECAR of hPSC-derived brown and white adipocytes. The OCR and ECAR were determined using no cells (brown line) and cells differentiated with adipogenic media alone (untransduced) as controls. Cells in which PPARG2 was exogenously expressed (+PPARG2) are represented with a green graph, cells that were transduced with lenti-PPARG2 and lenti-CEBPB (+PPARG2-CEBPB) are shown with a black line and lenti-PPARG2, lenti-CEBPB and lenti-PRDM16 (+PPARG2-CEBPBPRDM16)-transduced cells are indicated by a blue line. The OCR and ECAR were measured over time in approximately 5 min intervals. The first two measurements were conducted to establish a baseline rate, followed by three measurements after the addition of oligomycin, an ATPase inhibitor (I). By uncoupling the proton gradient with CCCP, the maximum OCR and ECAR rates were determined over the next three time intervals (II). Finally, at two time points, measurements were conducted after inhibition of the mitochondrial respiratory chain with antimycin (III). All experiments were conducted with BJ-RiPSC-derived MPCs. $P$ values represent two-tailed Student's $t$-tests between untransduced and transduced cells. Values for each cell line, $n=4, * P<0.01$, $* * P<0.001$ (s.e.m.). 

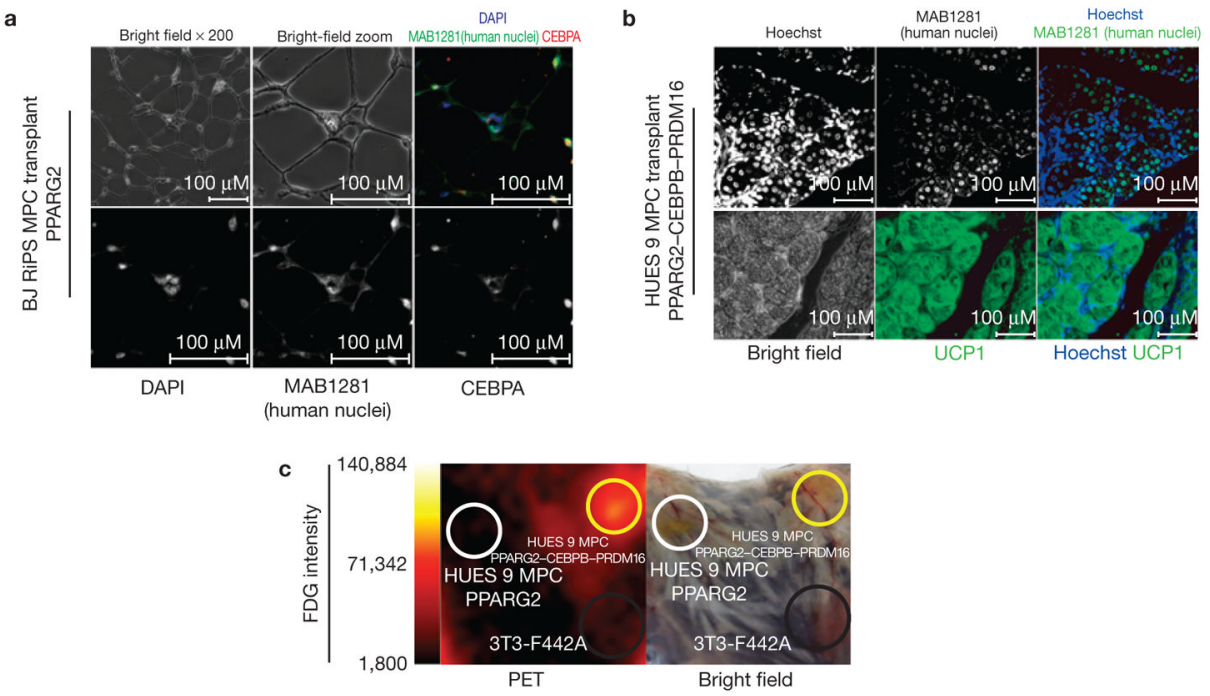

Figure 7.

Generation of functional hPSC-derived brown and white adipose tissue in vivo. (a) HUES-9-derived MPCs were transduced with lenti-PPARG2 and after 2 weeks of differentiation collected and injected subcutaneously into RAG2; IL2 $\gamma$ C double-knockout mice. Four to six weeks after the injection, prominent cell growth was visible at the injection site. This fat pad was collected, sectioned and stained. Top panels, bright-field morphology of the transplant sections (left); zoomed bright-field image of the transplant section (middle); immunohistochemistry overlay of stainings for nuclear marker DAPI (blue), staining with a human-specific nuclei marker MAB1281 (green) and staining with antibody against CEBPA (red; right). Bottom panels, staining with nuclear marker DAPI (left), staining with a human-specific nuclei marker MAB1281 (middle) and staining with antibody against CEBPA (right). (b) HUES-9-derived MPCs were transduced with a combination of lenti-PPARG2, lenti-CEBPB and lenti-PRDM16 and transplanted and collected as described above. Specimens were sectioned and adjacent slides were stained with UCP1 and MAB1261, a human-specific nuclei marker (all images $\times 200$ magnification). (c) Left, FDG intensity. Middle, PET image of mouse skin with transplants from HUES 9 MPC (PPARG2), HUES 9 MPC (PPARG2-CEBPB-PRDM16) and control 3T3-F442A cells. Right, the corresponding bright-field image of mouse skin with transplants from HUES 9 MPC (PPARG2), HUES 9 MPC (PPARG2-CEBPB-PRDM16) and control 3T3-F442A cells. 